\title{
Microvascular Decompression by Interposition Method for Treatment of Trigeminal Neuralgia Due to Vertebrobasilar Dolichoectasia: A Retrospective Single-Center Study
}

Feng Yu ( $\square$ yufeng.yf@163.com )

PLA 960th Hospital https://orcid.org/0000-0002-5991-9981

Jia Yin

PLA 960th Hospital

Pei-gang Lu

PLA 960th Hospital

Zhen-yu Zhao

PLA 960th Hospital

Yong-qiang Zhang

PLA 960th Hospital

Xue-zhong Men

PLA 960th Hospital

\section{Research Article}

Keywords: Trigeminal neuralgia, vertebrobasilar dolichoectasia, microvascular decompression, vasculonervous conflict

Posted Date: December 28th, 2021

DOI: https://doi.org/10.21203/rs.3.rs-1180077/v1

License: (c) (i) This work is licensed under a Creative Commons Attribution 4.0 International License. Read Full License

Version of Record: A version of this preprint was published at Neurosurgical Review on April 7th, 2022. See the published version at https://doi.org/10.1007/s10143-022-01776-6. 


\section{Abstract}

Trigeminal neuralgia (TN) due to vertebrobasilar dolichoectasia (VBD) is a rare disease that can be challenging to treat. The objectives of this study are to investigate the characteristics of patients with TN due to VBD and to analyze the efficacy of microvascular decompression (MVD) by the interposition method for treatment of the condition. From 2010 till 2020, the data of 30 patients with TN due to VBD who were treated with MVD by the interposition method were analyzed retrospectively. The characteristics of the patients were compared with those of patients with non-VBD TN $(n=815)$. Kaplan-Meier survival analysis was performed to determine pain-free survival. The 30 patients $(21$ males, 9 females; mean age, 63.03 years) accounted for $3.55 \%$ of all patients with TN during the study period. In 30 patients, the offending vessel was the basilar artery (BA) in 1 patient, the vertebral artery (VA) in 6 patients, the VA plus the superior cerebellar artery (SCA) in 6 patients, the VA plus the anterior inferior cerebellar artery (AICA) in 12 patients, and the VA+SCA+AICA in 5 patients. Compared to non-VBD TN patients, those with TN due to VBD were significantly more likely to be male, to have TN of the left side, and to have hypertension (all $\mathrm{P}<$ 0.001). Mean age at surgery $(P=0.057)$ and symptom duration $(P=0.308)$ were comparable between the two groups. All 30 patients had immediate relief of facial pain after MVD and could stop medication. There were no postoperative complications. Over mean follow-up of 76.67 months, 3 patients had recurrence. The mean duration of pain-free survival was 70.77 months. In conclusions, TN due to VBD appears to be more likely in males, in those with hypertension, and to involve the left side. The interposition method performed by experienced and skilled neurosurgeons is a safe and effective treatment for TN due to VBD. Further studies are needed to analyze the associated long-term results and the pain recurrence rate among this special population.

\section{Introduction}

Vertebrobasilar dolichoectasia (VBD), first described by Smoker, is a rare cerebrovascular disease characterized by ectatic, elongated, and tortuous vertebrobasilar arteries (VBA)[24]. In angiographic and autopsy studies, the prevalence of VBD is less than $0.05 \%[5,8]$. A study from Japan found asymptomatic VBD in $1.3 \%$ of patients undergoing magnetic resonance imaging (MRI) or magnetic resonance angiography (MRA) examinations for various reasons[13]. The ectatic elongated arteries may sometimes compress the root of the trigeminal nerve or facial nerve and cause trigeminal neuralgia (TN) and/or hemifacial spasm. Impingement on the trigeminal nerve root is less likely because of the anatomy of the region. TN due to VBD accounts for only about $2 \%-7.7 \%$ of all cases of $T N[7,15,19,20,25,26,29,30]$. Surgical treatment can be challenging as the tortuous VBAs are difficult to separate from the nerve root in the narrow space of the cisterns. Microvascular decompression (MVD) is the treatment of choice because it is nondestructive and addresses the vascular etiology. There are two methods to deal with the offending VBA during MVD: the interposition method and the transposition method. The former, which is the traditional method, involves insertion of an implant between the offending vessel and the nerve, while the latter method involves repositioning of the VBA using clips, biomedical glues, or other measures. Both methods have advantages and disadvantages. Several authors have found the interposition method to 
be simple and effective[1, 7, 25, 27, 29, 31], but others believe that transposition method-although more risky-is more efficacious and also avoids the complications associated with the interposition method (e.g., adhesion and granuloma formation)[10, 12, 14, 16, 20, 28].

At our hospital, over the last 10 years, we have used the interposition method to treat 30 patients with TN due to VBD. The aim of this study is to describe the characteristics of the patient, our surgical technique, and the long-term outcomes of treatment.

\section{Materials And Methods}

Between 2010 and 2020, out of 845 patients presenting with TN at our center, 30 (3.55\%) patients were diagnosed to have TN due to VBD and were treated by the interposition technique. Among of them, 2 patients coexisted ipsilateral hemifacial spasm and the rate of associated hemifacial spasm was $6.67 \%$. The demographic and clinical data of these patients were collected from the hospital records and retrospectively analyzed. All patients underwent high-resolution MRI and computed tomography (CT) scans and MRA preoperatively. To clearly define the structure of the vessels, three-dimensional time-offlight and fast imaging employing steady-state acquisition sequences were adopted. In all patients, the imaging features satisfied the VBD diagnostic criteria. There was a clear relationship between the VBA and the trigeminal nerve in all 30 patients (Figure 1A-D).

\section{Mvd Procedure}

With the patient in the lateral decubitus position, retrosigmoid suboccipital craniotomy was performed. The arachnoid membrane around the trigeminal nerve was opened to reveal the tortuous vertebral arteries situated ventral to the caudal cranial nerves (Figure 2A, C). The degree of vasculonervous conflict (VNC) varied: it was classified as grade I if the vessel was in contact with the nerve root but there was no visible indentation; as grade II (the most frequent) if there was displacement and/or distortion of the root; and as grade III if there was marked indentation of the nerve root[22]. To decompress the trigeminal nerve, shredded Teflon sponge was introduced piece-by-piece between the VBA complex and the trigeminal nerve and the medulla oblongata to obtain progressive mobilization (Figure 2B, D).

\section{Clinical Evaluation}

Therapeutic efficacy was assessed using the Barrow Neurological Institute (BNI) pain intensity score: BNI score I indicates no pain, without need for any medication; BNI score II indicates occasional pain, but no need for medication; BNI score III indicates some pain that is adequately controlled with medication; BNI score IV indicates some pain, not adequately controlled with medications; and BNI score $\mathrm{V}$ indicates severe pain, not relieved by medication[21]. 
Immediate postoperative outcome was classified as "complete pain relief" (BNI score I), "adequate pain relief" (BNI score I and II), or "treatment failure" (BNI score IV and V). Long-term follow-up was by telephonic or in-person interviews. Recurrence was defined as a return to preoperative pain status after achieving adequate pain relief (BNI score I-III).

The study was approved by the institutional review board of our hospital, with waiver of the need for consent.

\section{Statistical analysis}

Data analysis was with SPSS 20 (IBM Corp., Armonk, NY, USA). Age at surgery, duration of symptoms, sex distribution, side affected, and prevalence of hypertension were compared between patients with TN due to VBD and patients with non-VBD TN. The $t$ test was used to compare continuous data between groups and the chi-square test to compare categorical data. $P<0.05$ was considered statistically significant. Pain free survival was defined as the time to recurrence of facial pain after surgery. Kaplan-Meier curves were constructed based on pain free survival.

\section{Results}

Of the 845 patients with TN treated at our hospital from 2010 to 2020, 30 had TN due to VBD and 815 had non-VBD TN; Table 1 lists the characteristics of patients in the two groups. Compared to patients with non-VBD TN, patients with TN due to VBD were significantly more likely to be males (70\% vs. $37.7 \%$, $P<0.001)$, with a trend toward older patients $(P=0.057)$; to have $T N$ of the left side $(73.3 \%$ vs. $36.9 \%, P<$ $0.001)$; and to have hypertension ( $73 \%$ vs. $25.9 \%, P<0.001)$.

Table 1

Comparison of characteristics between patients with non-VBD TN and with TN due to VBD

\begin{tabular}{|llll|}
\hline & Non-VBD TN, $\mathbf{n}=815$ & TN due to VBD, $\mathbf{n}=30$ & $\mathrm{P}$ \\
\hline Age at surgery (years) & $59.23 \pm 10.83$ & $63.03 \pm 7.55$ & 0.057 \\
\hline Symptom duration (months) & $69.72 \pm 25.29$ & $56.13 \pm 29.57$ & 0.308 \\
\hline Males $(\mathrm{M})$ & $307(37.7 \%)$ & $21(70 \%)$ & $<0.001$ \\
\hline Side $(\mathrm{L})$ & $301(36.9 \%)$ & $22(73.3 \%)$ & $<0.001$ \\
\hline Hypertension ${ }^{+}$ & $211(25.9 \%)$ & $22(73 \%)$ & $<0.001$ \\
\hline Data are means \pm SD or $\mathrm{n}(\%)$ & & \\
\hline TN: Trigeminal neuralgia; VBD: vertebrobasilar dolichoectasia & \\
\hline${ }^{+}$Systolic blood pressure $\geq 140$ mmHg and/or diastolic blood pressure of $\geq 90 \mathrm{mmHg}$ \\
\hline
\end{tabular}




\section{Surgical Findings}

Table 2 lists the surgical findings (offending vessels and degree of VNC), pain distribution, and BNI scores in patients with TN due to VBD. While only one branch of the trigeminal nerve was affected in 7 patients, two branches were affected in 20 patients, and all three branches were affected in 3 patients. The offending vessel was the basilar artery (BA) in 1 patient, the vertebral artery (VA) in 6 patients, the VA plus the superior cerebellar artery (SCA) in 6 patients, the VA plus the anterior inferior cerebellar artery (AICA) in 12 patients, and the VA+SCA+AICA in 5 patients. Degree I VNC was seen in 1 patient, degree II VNC in 17 patients, and degree III VNC in 12 patients. 
Table 2

Operative findings and outcomes in the 30 patients with trigeminal neuralgia due to vertebrobasilar dolichoectasia

\begin{tabular}{|c|c|}
\hline Findings & $\mathrm{n}(\%)$ \\
\hline \multicolumn{2}{|l|}{ Offending vessel } \\
\hline VA & $6(20 \%)$ \\
\hline BA & $1(3.3 \%)$ \\
\hline VA+SCA & $6(20 \%)$ \\
\hline $\mathrm{VA}+\mathrm{AICA}$ & $12(4 \%)$ \\
\hline$V A+S C A+A I C A$ & $5(16.7 \%)$ \\
\hline Degree of VNC & $1(3.3 \%)$ \\
\hline \multicolumn{2}{|l|}{ ] } \\
\hline प & $17(56.7 \%)$ \\
\hline प & $12(40 \%)$ \\
\hline Pain distribution & $0(0 \%)$ \\
\hline $\mathrm{V} 1$ & $6(20 \%)$ \\
\hline $\mathrm{V} 2$ & $1(3.3 \%)$ \\
\hline V3 & $5(16.7 \%)$ \\
\hline $\mathrm{V} 1+2$ & $15(50 \%)$ \\
\hline $\mathrm{V} 2+3$ & $3(10 \%)$ \\
\hline$V 1+2+3$ & $30(100 \%)$ \\
\hline BNI score immediately after surgery & $0(0 \%)$ \\
\hline 口 & $27(90 \%)$ \\
\hline $\mathbb{Q}-\mathbb{Q}$ & $3(10 \%)$ \\
\hline \multicolumn{2}{|l|}{ BNI score at last follow-up } \\
\hline \multicolumn{2}{|l|}{ I } \\
\hline \multicolumn{2}{|l|}{ IV } \\
\hline
\end{tabular}

\section{Surgical Outcomes}


Following MVD, all 30 patients had complete relief of facial pain and could stop medication. No obvious postoperative complications (facial numbness, facial palsy, hearing loss, or diplopia) occurred in any patient. There were no deaths. Patients were followed up for periods ranging from 16 months to 130 months (mean, 76.67 months). Pain recurred in three patients (at 7, 12, and 52 months post operation); two of these patients underwent percutaneous radiofrequency thermocoagulation for pain relief, while one patient underwent repeat MVD by the interposition procedure.

\section{Pain-free Survival}

Of the 30 patients included in the Kaplan-Meier survival analysis, 3 patients had pain recurrence (at 7, 12 , and 52 months post-operation, respectively). The mean duration of pain-free survival was 70.77 months (Figure 3).

\section{Discussion}

\section{Cause of VBD}

The pathogenesis of VBD is not clear, and many factors are probably involved. The vertebral arteries arise from the subclavian artery. While the right subclavian artery arises from the brachiocephalic trunk, the left subclavian artery springs directly from the aortic arch. Therefore, blood flow and stress are higher in the left vertebral artery than the right one. Some scholars believe that atherosclerosis is the main cause of $\operatorname{VBD}[2,3]$; however, the relationship remains to be confirmed. Histopathologically, tears in the internal elastic lamina, smooth muscle atrophy, and reticular fiber deficiency have been demonstrated in the dolichoectatic arteries, suggesting that VBD may be caused by congenital dysplasia in some patients[9, $11,18]$.

\section{Safety Of Interposition Method}

MVD is the treatment of choice for TN caused by VBD. The interposition method and the transposition method both aim to separate the offending vessel from the nerve. We preferred to use the interposition technique in our patients for two reasons. First, interposition of Teflon patches between the root entry zone and the offending vessels is relatively easily performed. Once the tense trigeminal nerve is loosened, further attempts to mobilize the VBA complex are not necessary. Meanwhile, transposition of the large and tortuous VBA is often not easy due to the large size and poor elasticity of the arteries, and the difficulty of surgical manipulation within the narrow available space. There is also the risk of chemical vasculitis and adhesion of neurovascular structures with use of glues, or of avulsion of perforators to the brainstem due to aggressive movement of the VBA complex. If multiple vessels are involved, the manipulation becomes even more difficult and risky. 


\section{Clinical Outcomes}

Excellent immediate outcomes were achieved with the interposition method in all 30 of our patients. Only 3 patients had recurrence (over mean follow-up of 76.67 months) and the recurrence rate is $10 \%$. The current literature reports immediately pain relief in $90 \%$ patients and a successful outcome at 15 years of $75 \%[23]$.However, the specific efficacy of MVD in case of VBD is rarely evaluated. A random-effects metaanalysis between 1990 and 2020, which including 167 patients with TN caused by VBD, demonstrated that the rate of pain relief immediately after surgery is approximately $97 \%$. At last follow-up (55 months), this rate decreased to $92.9 \%$ confirming MVD as effective treatment for TN due to VBD[6]. This result is similar to our recurrence rate in consideration of an average TN recurrence rate of $3.5 \%$ per year [4]. We can identify three reasons for the excellent results with MVD in our patients. First, the offending vertebrobasilar vessel complex was usually obvious intraoperatively and easily identified by the operator; second, the VNC was mostly grade II or III, the effect of MVD is superior to TN caused by undefined vessels; and third, the narrow space made it difficult for other vessels to translocate to the root entrance/exit zone after the VBA complex was moved.

Age at surgery and mean preoperative symptom duration were not significantly different between patients with TN due to VBD and patients with non-VBD TN at our hospital. However, in the study by Linskey[15], patients with TN due to VBD were older than patients with non-VBD TN.

\section{Repeat Mvd}

To the best of our knowledge, repeat MVD after failure of the first treatment for TN due to VBD has not been reported previously; one patient in our series needed a repeat procedure for pain relief. According to a previous study, Teflon granuloma is the main reason for pain recurrence.[17] Surgical removal of the granuloma combined with repeat of the MVD is the preferred treatment. In our patient, the previously planted Teflon had not become stiff and knotted, also without Teflon granuloma, and there was impingement by a new VNC (the SCA). We excised part of previously inserted Teflon sponge, and introduced shredded Teflon sponge piece-by-piece between the SCA and the trigeminal nerve and brain stem. The patient became pain-free and off medication again, although there was short period of facial numbness after the repeat surgery.

\section{Study Limitations}

Our study has some limitations. First, the sample size is small because of the relative rarity of the pathology; thus, statistical analysis may be somewhat underpowered. Second, this was a nonrandomized, retrospective single-center study, so substantial bias is likely. Finally, postoperative follow-up in some patients was relatively short. Despite these limitations, this study provides insight into the postoperative pain-free survival and repeat MVD in patients with TN due to VBD. 


\section{Conclusions}

Patients with TN due to VBD appear more likely to be males, with a trend toward older patients, with hypertension, and to involve the left side. Surgical management of those patients is challenging. In the hands of experienced neurosurgeons, microvascular decompression by the interposition method can provide excellent outcomes in patients with trigeminal neuralgia due to vertebrobasilar dolichoectasia. Major complications, such as cerebellar hemorrhage or swelling, permanent facial numbness, hearing impairment were not encountered.

\section{Declarations}

Data availability May be considered upon request.

\section{Compliance with ethical standards}

Conflicts of interest The authors declare that they have no conflict of interest.

Ethical approval Research protocol was approved by the Institutional Review Board of PLA $960^{\text {th }}$ hospital.

Informed consent Informed consent was provided before intervention by each patient and his/her nearest family member.

Code availability Not applicable.

Funding This work was funded by the National Nature Science Foundation of China (No.81671201), and the PLA 960th hospital provided finical support in the form of a Research fund program grant (No.2017ZX04).

Credit Author Statement Feng yu: Resources, Conceptualization, Methodology, Writing-Original draft preparation.

Jia Yin: Funding acquisition,writing- Reviewing and Editing.

Pei-gang Lu: Conceptualization, Writing- Reviewing.

Zhen-Yu Zhao: Resources, Funding acquisition.

Yong-qiang Zhang: Data curation, Investigation.

Xue-zhong Men: Software, Validation.

\section{ORCID}

Feng Yu http://orcid.org/0000-0002-5991-9981 


\section{References}

1. Arrese I, Sarabia R (2016) Microvascular decompression for trigeminal neuralgia secondary to vertebrobasilar dolichoectasia. Case report, literature review, and pooled case analysis. Neurocirugía 27:304-309

2. Baquero M, Yayá-Huamán R (1998) Dolicoectasia vertebrobasilar. Rev Neurol 26:143-148

3. Boeri R, Passerini A (1964) The Megadolichobasilar Anomaly. J Neurol Sci 1:475-484

4. Burchiel KJ, Clarke H, Haglund M, Loeser JD (1988) Long-term efficacy of microvascular decompression in trigeminal neuralgia. J Neurosurg 69:35-38

5. Casas PI, Abruzzi M, Lehkuniec E, Schuster G, Muchnik S (1995) Dolichoectatic intracranial arteries. Advances in images and therapeutics. Medicina 55:59

6. Di Carlo DT, Benedetto N, Marani W, Montemurro N, Perrini P (2021) Microvascular decompression for trigeminal neuralgia due to vertebrobasilar artery compression: a systematic review and metaanalysis. Neurosurg Rev

7. El-Ghandour NM (2010) Microvascular decompression in the treatment of trigeminal neuralgia caused by vertebrobasilar ectasia. Neurosurgery 67:330-337

8. Flemming KD, Wiebers DO, Brown RD Jr, Link MJ, Huston J 3, McClelland RL, Christianson TJ (2005) The natural history of radiographically defined vertebrobasilar nonsaccular intracranial aneurysms. Cerebrovasc Dis 20:270-279

9. Greitz T, Löfstedt S (1954) The relationship between the third ventricle and the basilar artery.Acta radiologica:85-100

10. Grigoryan YA, Sitnikov AR, Grigoryan GY (2016) Trigeminal neuralgia and hemifacial spasm associated with vertebrobasilar artery tortuosity. Zhurnal voprosy neirokhirurgii imeni N. N Burdenko 80:44-56

11. Hegedus K (1985) Ectasia of the basilar artery with special reference to possible pathogenesis. Surg Neurol 24:463-469

12. Honey CM, Kaufmann AM (2018) Trigeminal Neuralgia due to Vertebrobasilar Artery Compression. World Neurosurg 118:e155-e160

13. Ikeda K, Nakamura Y, Hirayama T, Sekine T, Nagata R, Kano O, Kawabe K, Kiyozuka T, Tamura M, Iwasaki Y (2010) Cardiovascular risk and neuroradiological profiles in asymptomatic vertebrobasilar dolichoectasia. Cerebrovasc Dis 30:23-28

14. Lin CF, Chen HH, Hernesniemi J, Lee CC, Liao CH, Chen SC, Chen MH, Shih YH, Hsu SP (2012) An easy adjustable method of ectatic vertebrobasilar artery transposition for microvascular decompression. Clin Neurol Neurosurg 114:951-956

15. Linskey ME, Jho HD, Jannetta PJ (1994) Microvascular decompression for trigeminal neuralgia caused by vertebrobasilar compression. J Neurosurg 81:1-9

16. Liu J, Chen Z, Feng T, Jiang B, Yuan Y, Yu Y (2019) Biomedical Glue Sling Technique in Microvascular Decompression for Trigeminal Neuralgia Caused by Atherosclerotic Vertebrobasilar 
Artery: A Description of Operative Technique and Clinical Outcomes. World Neurosurg 128:e74-e80

17. Liu J, Wu G, Xiang H, Liu R, Li F, Hei B, Qian W, Song H, Liu Z (2020) Long-Term Retrospective Analysis of Microvascular Decompression in Patients With Recurrent Trigeminal Neuralgia. Front Neurol 11:584224

18. Lou M, Caplan LR (2010) Vertebrobasilar dilatative arteriopathy (dolichoectasia). Ann N Y Acad Sci 1184:121-133

19. Ma X, Sun X, Yao J, Ni S, Gong J, Wang J, Li X (2013) Clinical analysis of trigeminal neuralgia caused by vertebrobasilar dolichoectasia. Neurosurg Rev 36:573-577. discussion 577-578

20. MIYAZAKI S, FUKUSHIMA T, TAMAGAWA T, MORITA A (1987) Trigeminal neuralgia due to compression of the trigeminal root by a basilar artery trunk. Neurologia medico-chirurgica 27:742748

21. Rogers CL, Shetter AG, Fiedler JA, Smith KA, Han PP, Speiser BL (2000) Gamma knife radiosurgery for trigeminal neuralgia: the initial experience of The Barrow Neurological Institute. Int J Radiat Oncol Biol Phys 47:1013-1019

22. Sindou M, Howeidy T, Acevedo G (2002) Anatomical observations during microvascular decompression for idiopathic trigeminal neuralgia (with correlations between topography of pain and site of the neurovascular conflict). Prospective study in a series of 579 patients. Acta Neurochir (Wien) 144:1-12. discussion 12-13

23. Sindou M, Leston JM, Le Guerinel C, Keravel Y (2009) [Treatment of trigeminal neuralgia with microvascular decompression]. Neurochirurgie 55:185-196

24. Smoker WR, Corbett JJ, Gentry LR, Keyes WD, Price MJ, McKusker S (1986) High-resolution computed tomography of the basilar artery: 2. Vertebrobasilar dolichoectasia: clinical-pathologic correlation and review. AJNR Am J Neuroradiol 7:61-72

25. Sun S, Jiang W, Wang J, Gao P, Zhang X, Jiao L, Liu W, Shu K, Lei T (2017) Clinical analysis and surgical treatment of trigeminal neuralgia caused by vertebrobasilar dolichoectasia: A retrospective study. Int J Surg 41:183-189

26. Vanaclocha V, Herrera JM, Martinez-Gomez D, Rivera-Paz M, Calabuig-Bayo C, Vanaclocha L (2016) Is There a Safe and Effective Way to Treat Trigeminal Neuralgia Associated with Vertebrobasilar Dolichoectasia? Presentation of 8 Cases and Literature Review. World Neurosurg 96:516-529

27. Wang X, Wang H, Chen S, Liang H, Wang H, Xu M, Xu L (2019) The long-term clinical outcomes of microvascular decompression for treatment of trigeminal neuralgia compressed by the vertebrabasilar artery: a case series review. BMC Neurol 19:217

28. Xue F, Shen Z, Wang Y, Kwok SC, Yin J (2021) Microvascular decompression for hemifacial spasm involving the vertebral artery: A modified effective technique using a gelatin sponge with a FuAiLe medical adhesive. CNS Neurosci Ther 27:857-861

29. Yang XS, Li ST, Zhong J, Zhu J, Du Q, Zhou QM, Jiao W, Guan HX (2012) Microvascular decompression on patients with trigeminal neuralgia caused by ectatic vertebrobasilar artery complex: technique notes. Acta Neurochir (Wien) 154:793-797. discussion 797 
30. Yuan YJ, Xu K, Luo Q, Yu JL (2014) Research progress on vertebrobasilar dolichoectasia. Int J Med Sci 11:1039-1048

31. Zhang YQ, Yu F, Zhao ZY, Men XZ (2019) Combined Hyperactive Dysfunction Syndrome of the Cranial Nerves: Analysis of 37 Cases and Literature Review. World Neurosurg 129:e650-e656

\section{Figures}
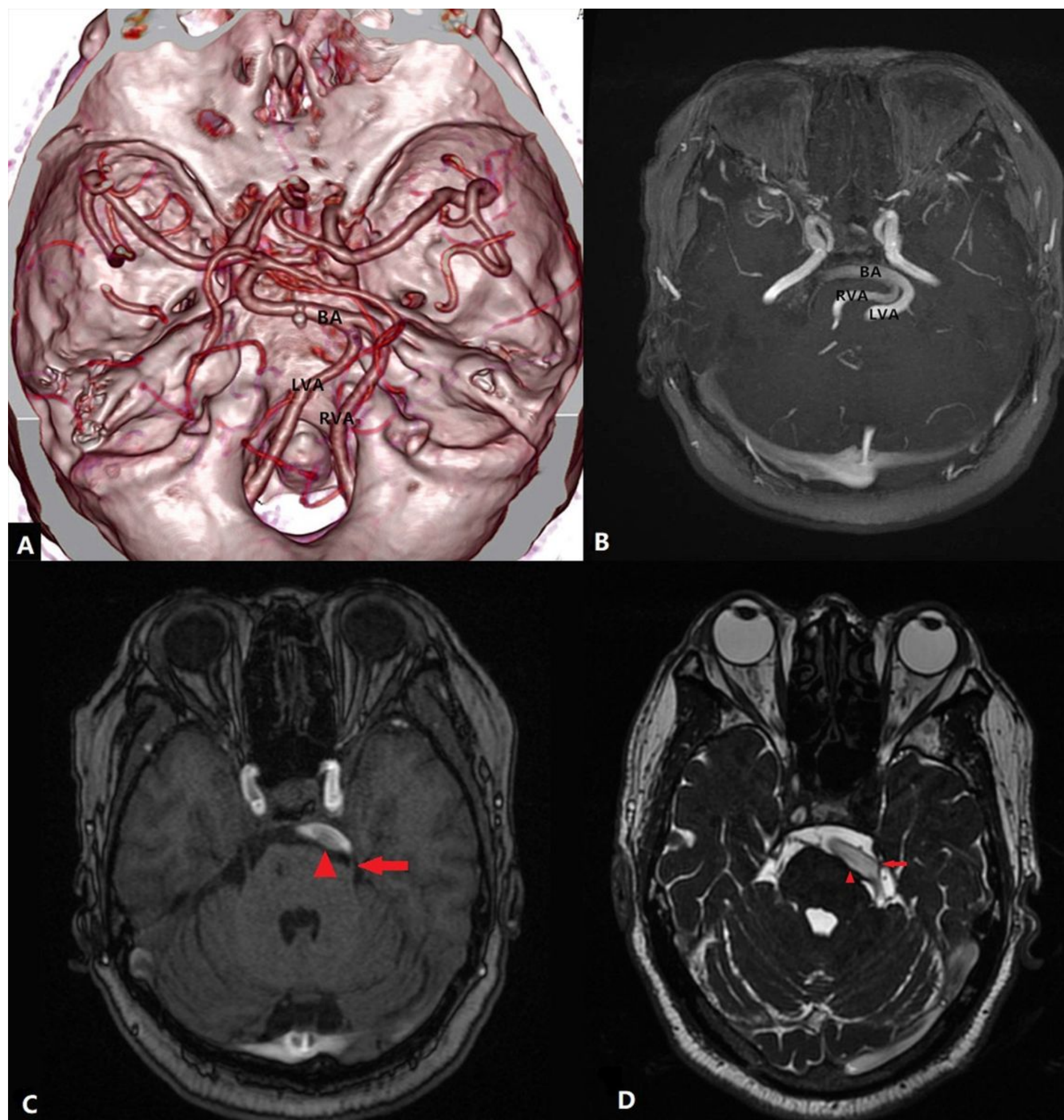

Figure 1 


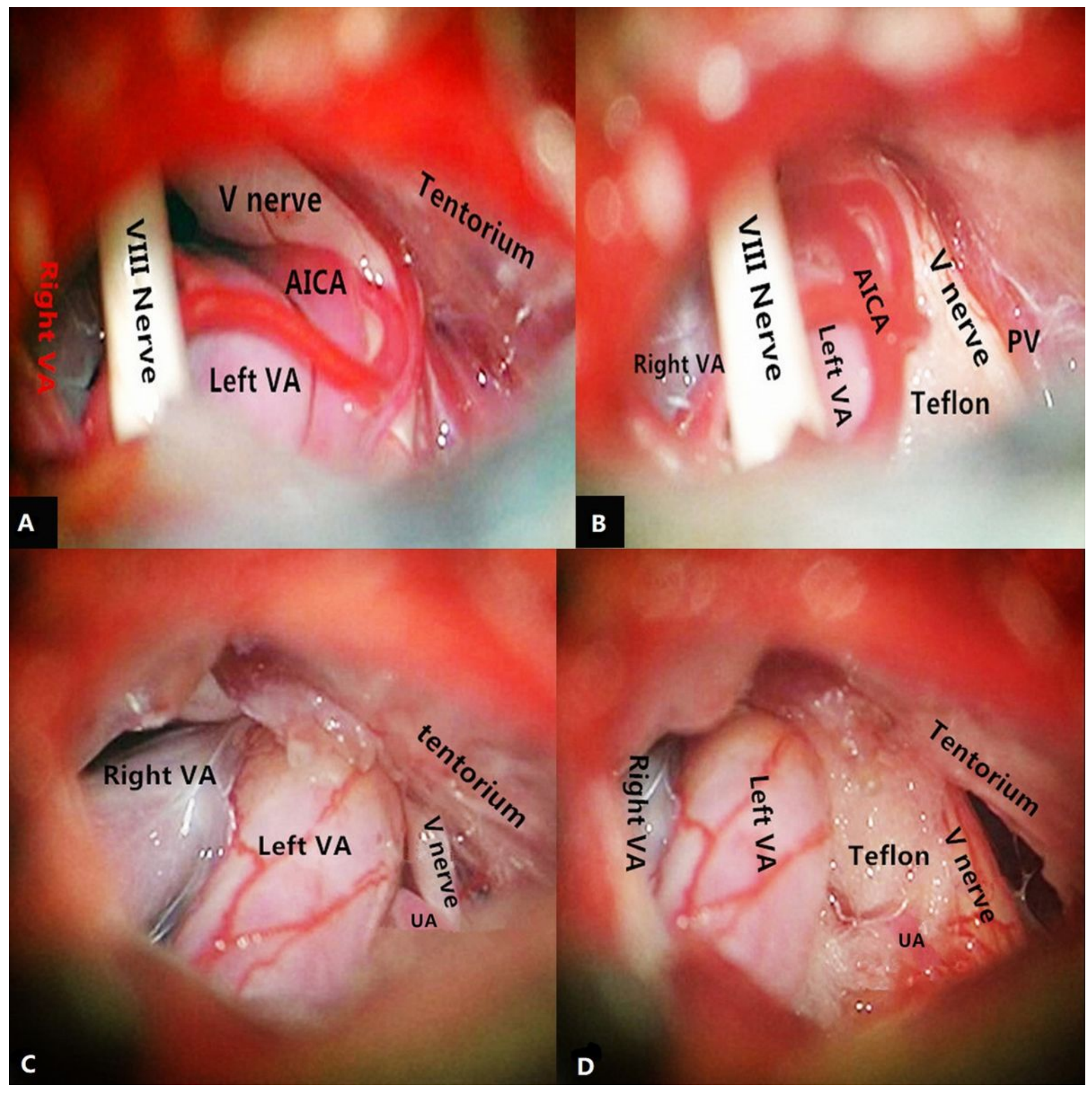

\section{Figure 2}

(a) Severe compression of the left trigeminal nerve against the tentorium by the ectatic, tortuous VA and AICA has resulted dislocation and distortion of the nerve. (b) A Teflon sponge was interposed between the VA and the trigeminal nerve. (c) The left vertebrobasilar dolichoectasia with atherosclerotic plaque is seen compressing the trigeminal nerve against the tentorium. (d) Pieces of shredded Teflon were introduced between the VBA and the trigeminal nerve to relieve the pressure on the trigeminal nerve. (VA: vertebral 
artery; PV: petrosal vein; AICA: anterior inferior cerebellar artery; UA: unnamed artery/arteriole; VBA: vertebrobasilar arteries)

\section{$\perp$ Survival function}

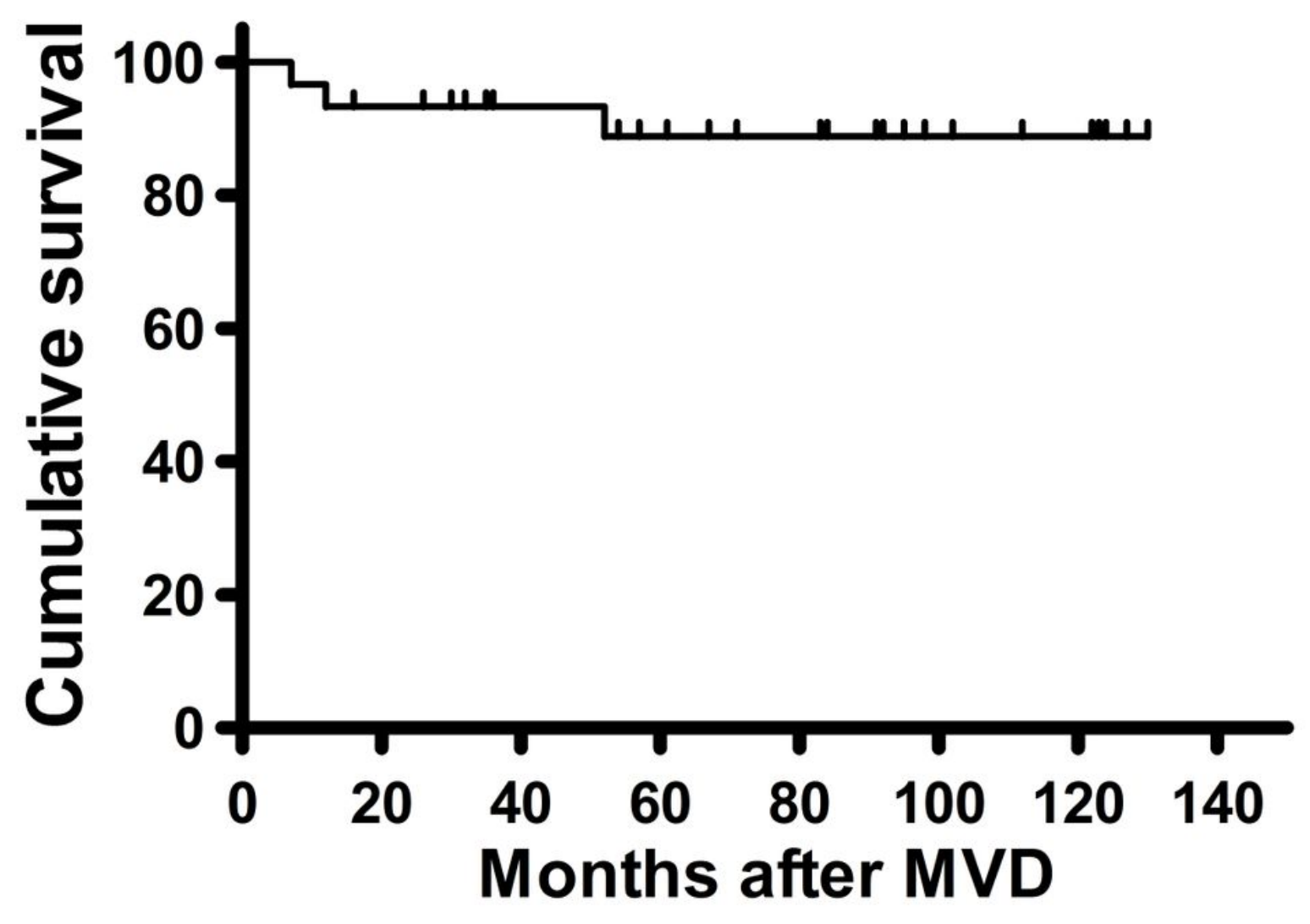

Figure 3

Kaplan-Meier survival analysis. Recurrence of pain occurred in three patients (at 7, 12, and 52 months post-operation). 